\title{
Biharmonic Attractors of Internal Gravity Waves
}

\author{
D. A. Ryazanov ${ }^{a, b}$, M. I. Providukhina ${ }^{c}$, I. N. Sibgatullin ${ }^{a, b, c, *}$, and E. V. Ermanyuk ${ }^{d}$ \\ ${ }^{a}$ Keldysh Institute of Applied Mathematics of the Russian Academy of Sciences, Moscow, Russia \\ ${ }^{b}$ Ivannikov Institute for System Programming of the Russian Academy of Sciences, Moscow, Russia \\ ${ }^{c}$ Shirshov Institute of Oceanology of the Russian Academy of Sciences, Moscow, Russia \\ ${ }^{d}$ Lavrentyev Institute of Hydrodynamics of the Siberian Branch of the Russian Academy of Sciences, \\ Novosibirsk, Russia \\ *e-mail:sibgat@ocean.ru
}

Received February 27, 2020; revised October 21, 2020; accepted December 1, 2020

\begin{abstract}
The hydrodynamic system that admits the development of internal wave attractors under biharmonic forcing is investigated. It is shown that in the case of low amplitude of external forcing the wave pattern consists of two attractors that interact between themselves only slightly: the total energy of the system is equal to the sum of energies of the components with high accuracy. In the nonlinear case the attractors interact in the more complex way which leads to the development of a cascade of triad interactions generating a rich set of time scales. In the case of closely adjacent frequencies of the components of a biharmonic perturbation, the nonlinear "beating" regime develops, namely, the mean energy of the system of coupled attractors performs oscillations at a large time scale that corresponds to the beating period. It is found that the high-frequency energy fluctuations corresponding to the same mean energy can differ by an order of magnitude depending on whether the envelope of the mean value increases or decreases.
\end{abstract}

Keywords: wave attractors, inertial waves, internal waves, wave turbulence

DOI: $10.1134 / \mathrm{S} 0015462821030046$

In a fluid with uniform density stratification the internal waves obey a specific dispersion relation [1] which connects the wave frequency and the angle of inclination of the wave beam with respect to the vector of gravity but does not contain the length scale. The consequence of the dispersion relation is the specific law of reflection from the boundaries under which the wave-ray angle with respect to the vertical, but not with respect to the normal to the surface, is conserved during reflection. In its turn, such a reflection law leads to the focusing of the wave beams due to reflection at inclined wall [2, 3]. In the presence of inclined walls in the closed volume of stratified fluid the wave focusing can lead to energy concentration along closed trajectories, the so-called wave attractors [4, 5]. Numerous studies reviewed in [3, 6] are devoted to the wave attractors. In geophysical applications, tidal currents are considered as the energy sources that generate the internal wave motions $[7,8]$. The interaction of the surface tides with the bottom topography leads to generation of internal tides; in this case, under certain conditions, the internal tidal motion can exist in form of attractors [9-12]. It is well known that the surface tides have a fairly complex spectrum and well-studied deterministic nature. The full-scale measurements show that the internal tides are distinguished by fairly lesser predictability. This results from both the high sensitivity of internal waves to the fine structure of stratification [13] and the development of instability [14, 15].

In order to investigate specifics of dynamics of the regimes with wave attractors, special methods of processing and visualizing the data, both experimental and numerical, were used. These methods include the time-and- frequency diagrams, the bi-spectra, the bi-correlations, and the Hilbert transforms with filtering the frequency range and the wave vectors $[16,17]$. The first attempts of two-dimensional numerical simulation demonstrated qualitative correspondence of the low-amplitude regimes of wave attractors [18, 19]. The investigation of three-dimensional effect of wave attractors carried out in [20,21] showed the adequate qualitative and quantitative correspondence of the results of direct numerical simulation and experiments and the difference between the results of previous two- and three-dimensional numerical calculations was explained. It should be noted that almost all the literature in the field of simulation of internal tides and wave attractors is devoted to the investigation of monochromatic external forcing [3, 6]. In the present study, the linear and nonlinear dynamics of stratified liquid are investigated numerically in 
(a)

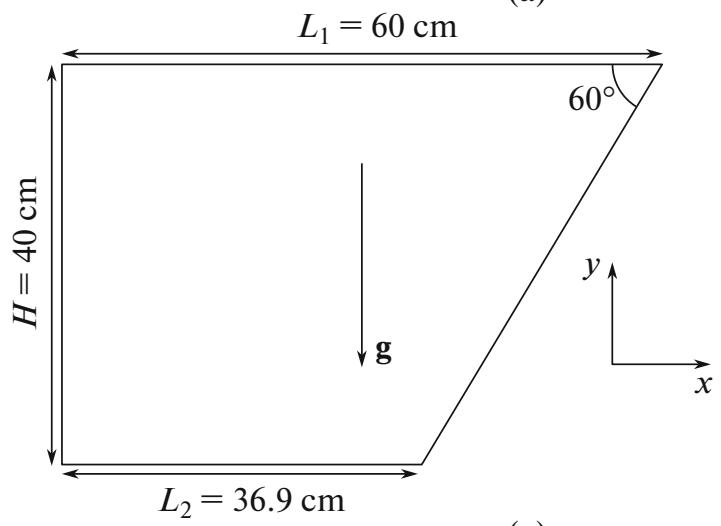

(c)

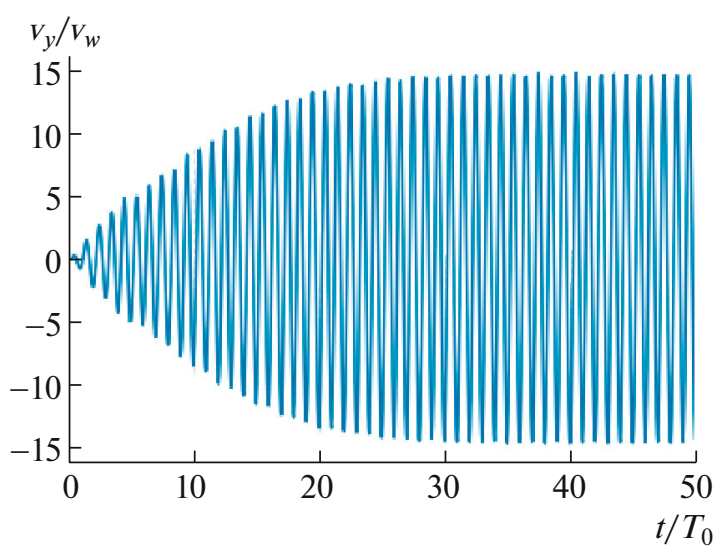

(b)

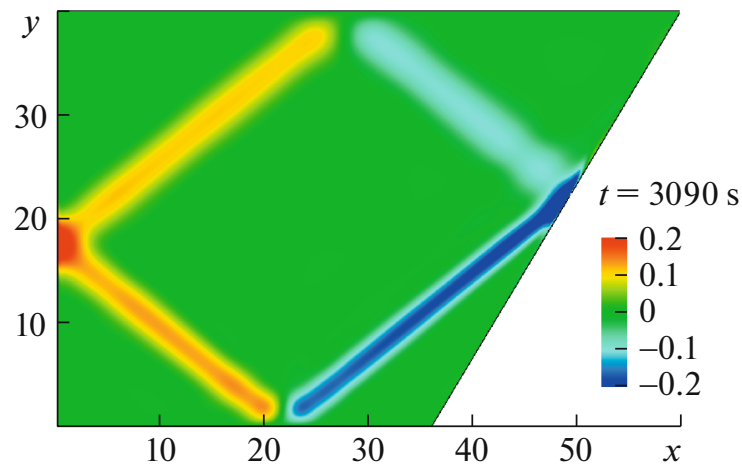

(d)

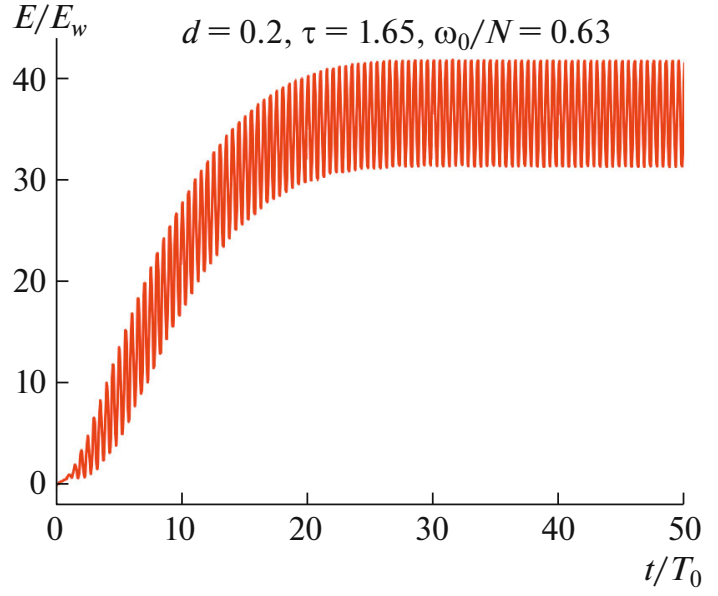

Fig. 1. Diagram of the computational domain (a); field of the vertical velocity component under the monochromatic impact at the amplitude $a=0.02 \mathrm{~cm}$ (b); corresponding graph of the vertical velocity in the middle of the first attractor ray as a function of time (c); kinetic energy as a function of time (d).

a model system which admits the existence of internal wave attractors under the impact of a biharmonic perturbation.

\section{MATHEMATICAL FORMULATION OF THE PROBLEM}

We will consider a tank of trapezoidal form with the greater base at top filled with a uniformly stratified liquid in the gravity field (Fig. 1). This geometric structure is fairly well studied in literature, namely, there is a detailed classification of the geometric configurations of attractors within the framework of the ray path theory [5], the stream functions are constructed within the framework of the inviscid liquid model [22], the linear [18] and nonlinear [23] scaling for the width of attractor's wave beams is investigated, and the energy cascade in the nonlinear regime is described [24]. The stratification can be characterized by the salinity gradient which is codirected with the vector of acceleration of gravity. In the initial state the buoyancy frequency

$$
N(y)=\sqrt{-\frac{g}{\rho(y)} \frac{d \rho(y)}{d y}}
$$

is constant over the entire tank. The no-slip conditions for the velocity $v_{n}=0$ and $v_{\tau}=0$ are fulfilled on all the boundaries (except for the upper boundary). For the salinity the insulation condition $\partial \rho_{s} / \partial n=0$ must be satisfied on all the boundaries. The vertical velocity component is specified on the upper boundary of the tank. This simulates the wave generator (wave maker) which performs harmonic oscillations in accordance with the following expressions:

for monochromatic oscillations $y(0, x, t)=a \sin \left(2 \pi x / L_{1}\right) \cos \left(\omega_{0} t\right)$;

for biharmonic oscillations $y(0, x, t)=a_{1} \sin \left(2 \pi x / L_{1}\right) \cos \left(\omega_{1} t\right)+a_{2} \sin \left(2 \pi x / L_{1}\right) \cos \left(\omega_{2} t\right)$. 
In what follows, we will consider the case $a_{1}=a_{2}=a$ as a model example. The small density drop over the tank depth $H$ makes it possible to use the Navier-Stokes equation in the Boussinesq approximation

$$
\begin{gathered}
\frac{\partial \vec{V}}{\partial t}+(\vec{V} \cdot \nabla) \vec{V}=-\frac{1}{\rho_{m}} \nabla \hat{p}+\mathrm{v} \Delta \vec{V}+\vec{f} \\
\frac{\partial \rho_{s}}{\partial t}+\vec{V} \cdot \nabla \rho_{s}=\nabla \cdot \frac{v}{\mathrm{Sc}}\left(\nabla \rho_{s}\right) \\
\nabla \cdot \vec{V}=0 .
\end{gathered}
$$

Here, $\vec{V}$ is the velocity vector with the components $\left\{v_{x}, v_{y}\right\}, v$ is the kinematic viscosity, $\rho_{m}$ is the density on the upper boundary, $\rho_{s}$ is a contribution to the density due to salinity, the reduced pressure $\hat{p}$ is the difference between the total and hydrostatic pressures for fresh water (at the density $\rho_{m}$ ), $\vec{f}=\rho_{s} / \rho_{m} \vec{g}$, $\mathrm{Sc}=\mathrm{V} / \kappa$ is the Shmidt number, where $\kappa$ is the diffusion coefficient.

In the linear regime the characteristic wave beam width is developed by the system as a result of balance between the effects of geometric focusing in reflection of the wave beam from the inclined wall and viscosity $[18,23]$. As the parameters characterizing the forcing, we can introduce the dimensionless frequency $\omega / N$ and the dimensionless amplitude $a / H$. In the numerical experiments the characteristic dimensionless amplitude is of the order of $O\left(10^{-3}\right)$, while the frequency $\omega / N$ is specified over the range of existence of the attractor whose beam "skeleton" has the shape of a parallelogram with a single point of reflection on each boundary. Following [5], the attractor of such a form will be called by the attractor of $(1,1)$ type. The degenerate forms of the $(1,1)$ attractor which correspond to the boundaries of its existence range are the trapezium diagonals. As a dimensionless parameter that characterizes the perturbation, we can introduce the Reynolds number determined from the maximum wave-maker velocity and the depth: $\operatorname{Re}=a \omega_{0} H / \mathrm{v}$. The present study is the continuation of the series of studies [20,23-25] on the experimental and numerical investigation of wave attractors in the laboratory-scale setups. For the sake of convenience in carrying out the comparisons, the numerical values of the parameters are given in the dimensionless and dimensional quantities.

In the two-dimensional formulation the numerical simulation of the system (1.1)-(1.3) was implemented in $[18,26]$ using the control volume approach and the qualitative agreement between the form of attractor in the calculation and linear theory was obtained. Approximately twofold difference between the amplitudes of wave motions was observed in quantitative comparison with the experiment. In [20, 24] the first three-dimensional simulation was carried out with the use of the spectral element method [27] and both the quantitative and qualitative agreement with the experiment was obtained.

All the following results were obtained from the numerical simulation of the nonlinear system of the Navier-Stokes equations in the Boussinesq approximation.

\section{MONOCHROMATIC PERTURBATION}

In the first stage of investigation, the monochromatic regimes were simulated with the aim to estimate the effectiveness of attractor generation under a given wave-maker amplitude and variation in the perturbation amplitude over the existence range of the attractor of $(1,1)$ type [5]. For the geometry shown in Fig. 1 the lower and upper boundaries of the existence range of the attractor of $(1,1)$ type correspond to $\omega_{\mathrm{cr} 1} / N=0.55$ and $\omega_{\mathrm{cr} 2} / N=0.74$, respectively. In reaching these critical frequencies, the parallelogram degenerates into a trapezium diagonal. As the integral dimensional measure of the effectiveness of attractor generation under constant wave-maker amplitude and invariable shape of the tank, we took the kinetic energy of liquid integrated over the trapezium area $S$

$$
E_{k}(t)=\int_{S} \frac{\rho_{m}}{2}\left[v_{y}^{2}(t)+v_{x}^{2}(t)\right] d S .
$$

For this measure we can introduce a value averaged over a sliding time window with the use of a fairly large number of the oscillation periods $\left\langle E_{k}(t)\right\rangle$ and variation with respect to the mean value calculated as follows: $r=D\left(E_{k}(t)-\left\langle E_{k}(t)\right\rangle\right) /\left\langle E_{k}(t)\right\rangle$, where $D\left(E_{k}(t)-\left\langle E_{k}(t)\right\rangle\right)$ is the variance with respect to the mean value. The dimensionless quantities $\bar{E}_{k}(t)$ and $\left\langle\bar{E}_{k}(t)\right\rangle$ are determined by dividing by $\rho_{m} S(a \omega)^{2} / 2$. It is well known that the regimes of motion in attractors can be similar to both the progressive and standing waves 
Table 1. Kinetic energy under the monochromatic impacts at the amplitude $a=0.02 \mathrm{~cm}$

\begin{tabular}{c|c|c|c}
\hline$\omega_{0} / N$ & $E_{k}$ & $\left\langle\bar{E}_{k}\right\rangle$ & $r$ \\
\hline $0.55\left(\omega_{\mathrm{cr}, 1}\right)$ & $1.32 \times 10^{-4}$ & 2.151 & 0.618 \\
0.58 & $8.45 \times 10^{-4}$ & 12.56 & 0.281 \\
0.59 & $12 \times 10^{-4}$ & 17.33 & 0.3 \\
0.63 & $29 \times 10^{-4}$ & 36.68 & 0.103 \\
0.641 & $23 \times 10^{-4}$ & 28.55 & 0.1129 \\
0.66 & $13.2 \times 10^{-4}$ & 15.14 & 0.152 \\
0.70 & $2.84 \times 10^{-4}$ & 2.896 & 0.295 \\
$0.74\left(\omega_{\mathrm{cr}, 2}\right)$ & $1.50 \times 10^{-4}$ & 1.356 & 0.215 \\
\hline
\end{tabular}

[25]. The quantity $r$ makes it possible to give the qualitative estimate of proximity of the regime observed to one of the limiting cases [25].

\subsection{Linear Regime}

In Fig. 1 we have reproduced the characteristic form of the dependences observed in the monochromatic regime at the low amplitude of the forcing for $a=0.02 \mathrm{~cm}\left(a / H=5 \times 10^{-4}\right)$ and $\omega / N=0.63$. The characteristic time of a transient to the stationary regime is of the order of 30 oscillation periods, the signal spectrum is monochromatic with high accuracy, and the oscillations of the kinetic energy with respect to the mean value have a low amplitude $(r=0.103)$. The beam with the maximum energy density appearing after focusing reflection from the inclined wall is notated as the first ray of attractor. In Table 1 we have given the integral parameters that characterize the linear monochromatic regimes at a given value of $a / H=5 \times 10^{-4}$ over the frequency range from $\omega_{\mathrm{cr} 1} / N=0.55$ to $\omega_{\mathrm{cr} 2} / N=0.74$. It can be seen that the kinetic energy of attractor is maximized at $\omega / N=0.63$ when the oscillation amplitude is kept constant. Obviously, at this value of the forcing frequency strong nonlinear effects should be expected with increase in the wave-maker oscillation amplitude. At $\omega / N=0.63$ the quantity $r$ reaches a minimum: in the attractor the motion is presented by the progressive wave.

\subsection{Nonlinear Regime}

In Fig. 2 we have reproduced the characteristic flow patterns and the dependences observed in the case of weakly nonlinear regime at $\omega / N=0.63$ for $a=0.05 \mathrm{~cm}\left(a / H=1.25 \times 10^{-3}\right)$. In the weakly nonlinear regime the triad resonance [15] takes place, two subharmonic daughter waves of low amplitude being generated. The time-frequency diagram shown in Fig. 2 represents the signal spectrum calculated in the sliding window and averaged over the neighborhood of a point lying in the middle of the first attractor branch. In this regime the frequency spectrum of internal waves is discrete with the predominant contribution that corresponds to the perturbation frequency $\omega_{0}$, two subharmonic frequencies $\omega_{1}^{*}+\omega_{2}^{*}=\omega_{0}$, two superharmonic frequencies $\omega_{1}^{* *}=\omega_{1}^{*}+\omega_{0}$ and $\omega_{2}^{* *}=\omega_{2}^{*}+\omega_{0}$, and the double frequency $2 \omega_{0}$.

The cascade of triad interactions develops with further increase in the perturbation amplitude to $a=$ $0.1 \mathrm{~cm}\left(a / H=2.5 \times 10^{-3}\right)$. In Fig. 3 we have reproduced the characteristic patterns of the wave fields, the spectra, and the time development of the oscillation process and the kinetic energy of the system. The discrete components that correspond to the frequencies of daughter waves appearing in the triad resonance, which are similar to the spectrum components appearing in the weakly nonlinear case $\left(a / H=1.25 \times 10^{-3}\right)$, predominate in the frequency signal spectrum. In this case the complete signal spectrum represents the superposition of the discrete and continuous spectra. The presence of the continuous spectrum testifies that the developed wave turbulence regime appears [24, 25]. In Table 2 we have given the corresponding characteristics for the kinetic energy of system in the strongly nonlinear regime. From a comparison of Tables 1 and 2 it can be seen that in the case of the developed wave turbulence regime the global dimensionless energy characteristics of the system (the average energy $\left\langle\bar{E}_{k}\right\rangle$ and variations with respect to the mean value $r$ ) differ only slightly from the dimensionless quantities characteristic of the linear regime. 
(a)

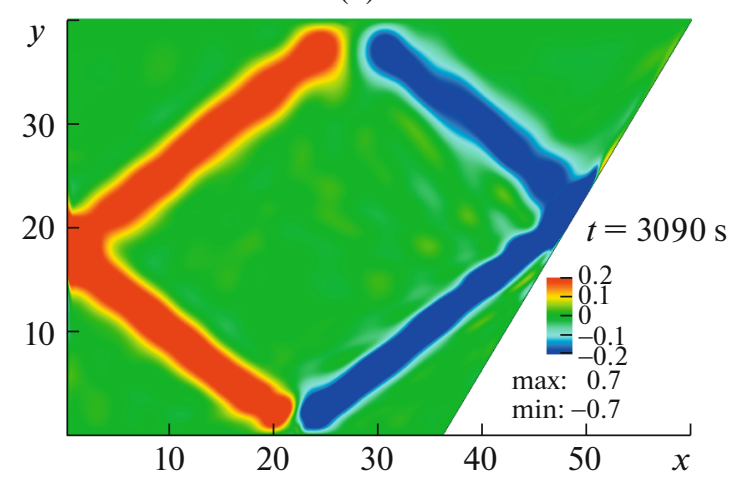

(c)

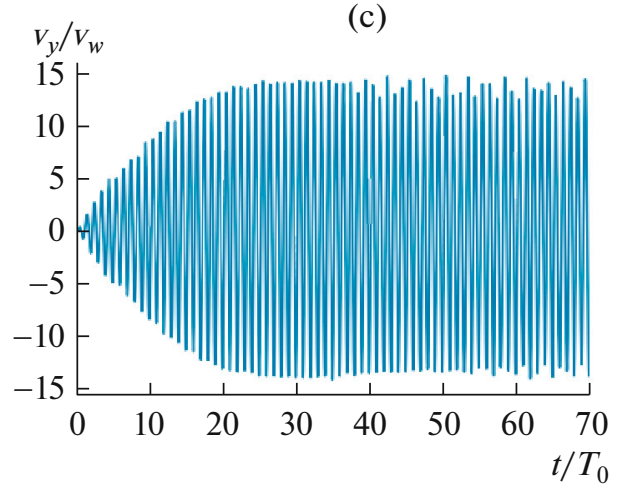

(e)

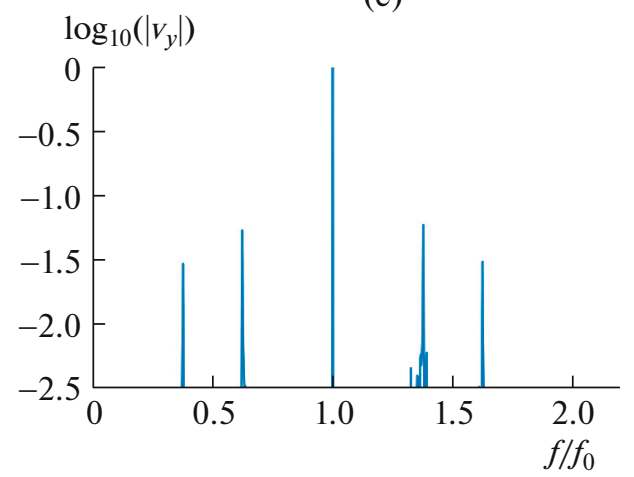

(b)

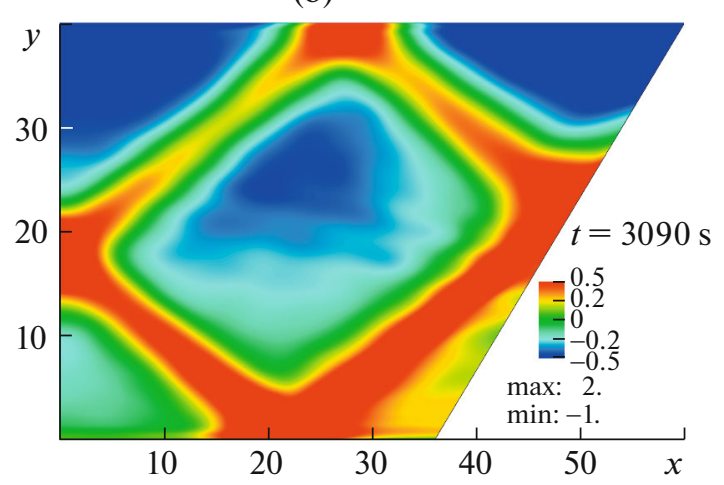

(d)
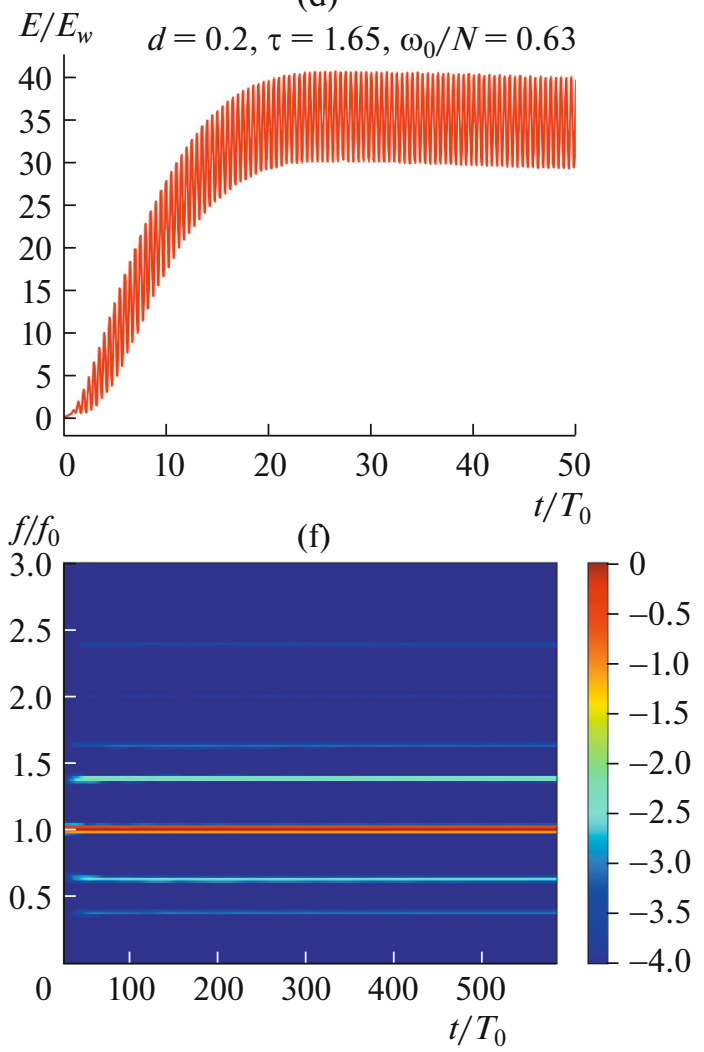

Fig. 2. Field of the vertical velocity component under the external monochromatic impact at the amplitude $a / H=$ $5 \times 10^{-4}$ (a) and the corresponding pressure field (b), dimensionless velocity in the middle of the first attractor ray (c), dimensionless kinetic energy (d), spectrum (e), and time-and-frequency diagram (f).

A comparison of the wave patterns in the linear and nonlinear cases shows that in the second case the energy is distributed more uniformly over the domain considered, namely, the attractor branches are wider and the daughter waves occupy the entire space.

\section{REGIMES WITH BIHARMONIC PERTURBATION}

\subsection{Linear Regime}

In Fig. 4 we have reproduced the characteristic example of the wave pattern and the main qualitative and quantitative characteristics of the system in the linear case under the biharmonic external forcing for following parameters: $\omega_{1} / N=0.58, \omega_{2} / N=0.66$, and $a=0.02 \mathrm{~cm}$. It can be seen that the system tends 
(a)

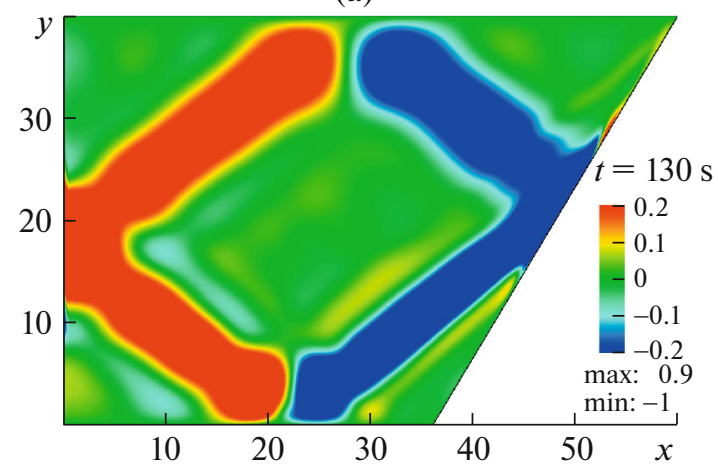

(c)

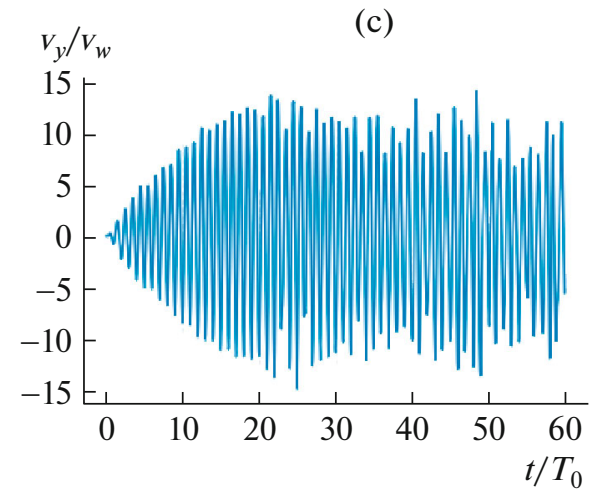

(e)

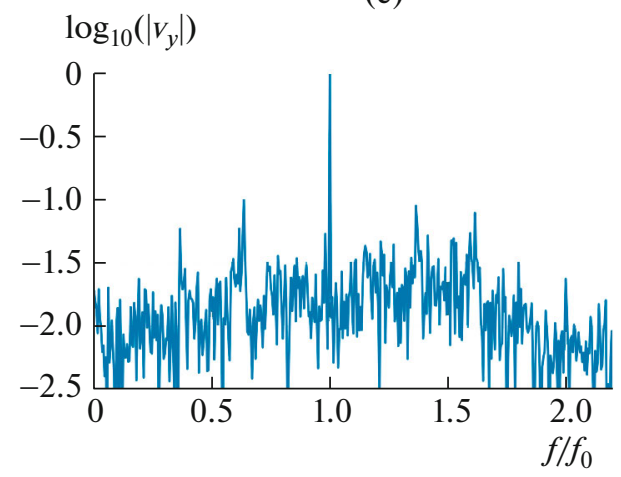

(b)

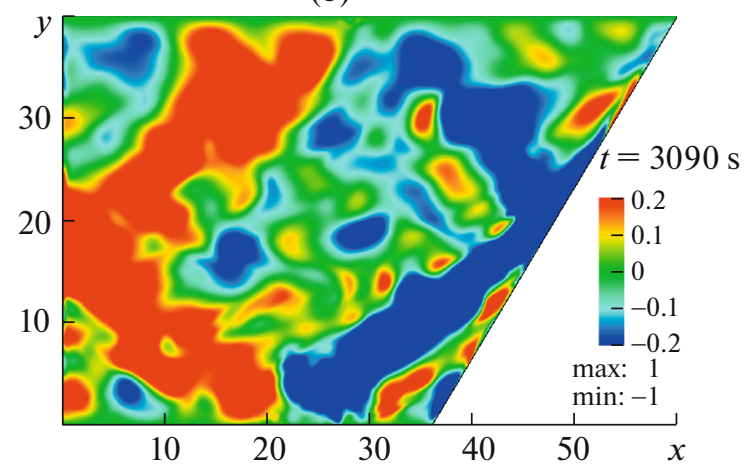

(d)
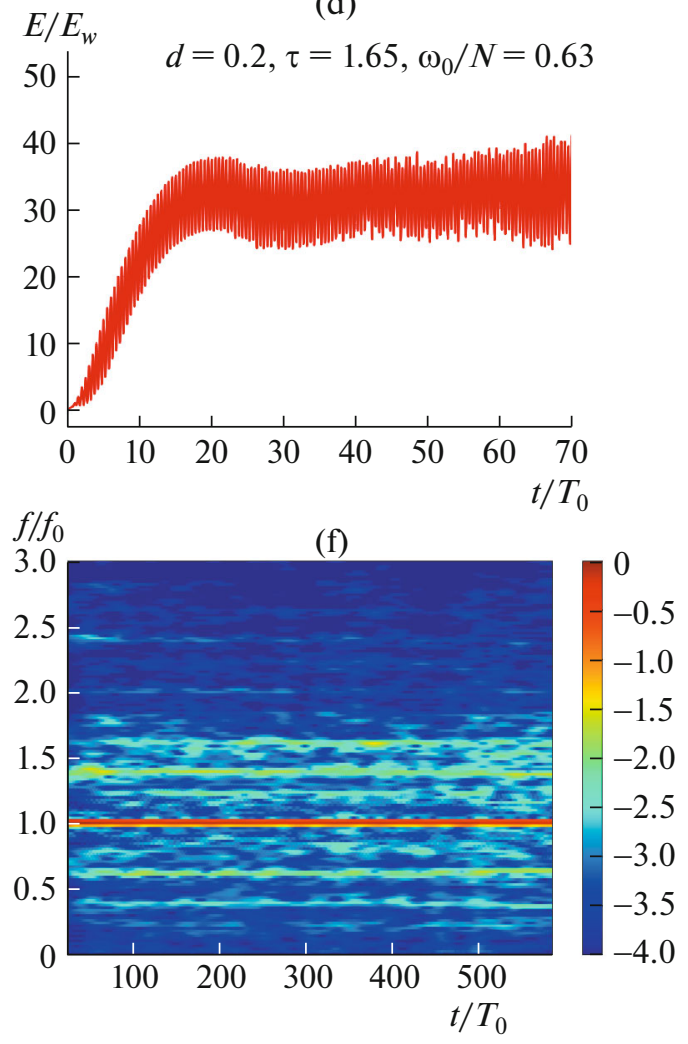

Fig. 3. Field of the vertical velocity component in the monochromatic regime at the amplitude $a=0.1 \mathrm{~cm} / \mathrm{s}$ during formation of an attractor (a) and after a cascade of instabilities (b), velocity in the middle of the first attractor ray as a function of time (c), kinetic energy as a function of time (d), frequency spectrum of the velocity in the wave turbulence regime (e), and time-and-frequency diagram (f).

to the quasi-steady-state beating regime at time-scale of the order of 40 oscillation periods. This is close to the characteristic time of transient to the process of steady-state oscillations in the monochromatic case. The peaks corresponding to the frequencies of external perturbation predominate over the frequency spectrum; there are also peaks corresponding to the frequency $2 \omega_{1} / N$ and the difference frequency

Table 2. Kinetic energy under the monochromatic impacts at the amplitude $a=0.1 \mathrm{~cm}$

\begin{tabular}{c|c|c|c}
\hline$\omega_{0} / N$ & $E_{k}(\mathrm{erg})$ & $\left\langle\bar{E}_{k}\right\rangle$ & $r$ \\
\hline $0.55\left(\omega_{\mathrm{cr}, 1}\right)$ & $33.0 \times 10^{-4}$ & 2.14 & 0.6193 \\
0.63 & $725 \times 10^{-4}$ & 36.7 & 0.1346 \\
$0.74\left(\omega_{\mathrm{cr}, 2}\right)$ & $37.0 \times 10^{-4}$ & 1.35 & 0.2192 \\
\hline
\end{tabular}


(a)

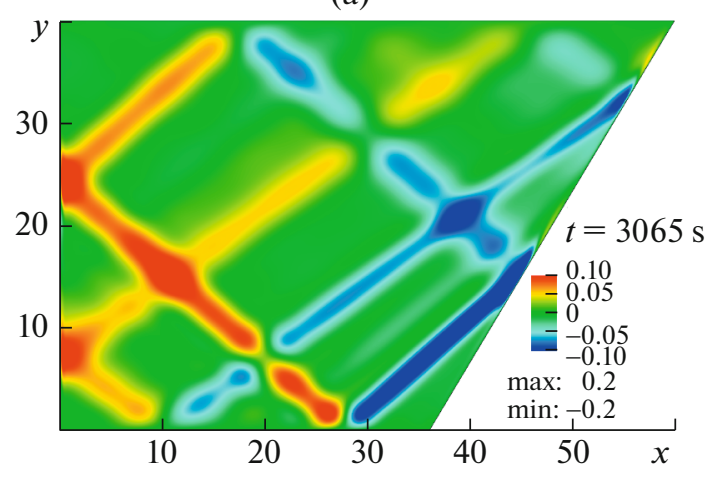

(c)

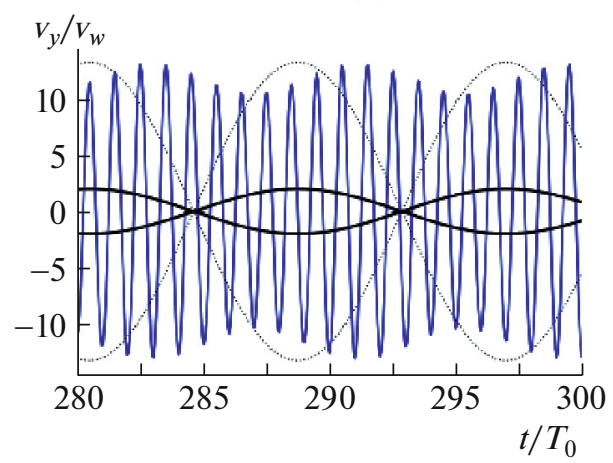

(e)

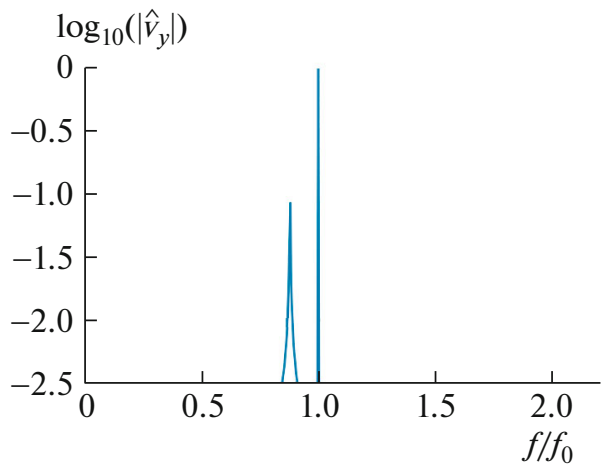

(b)

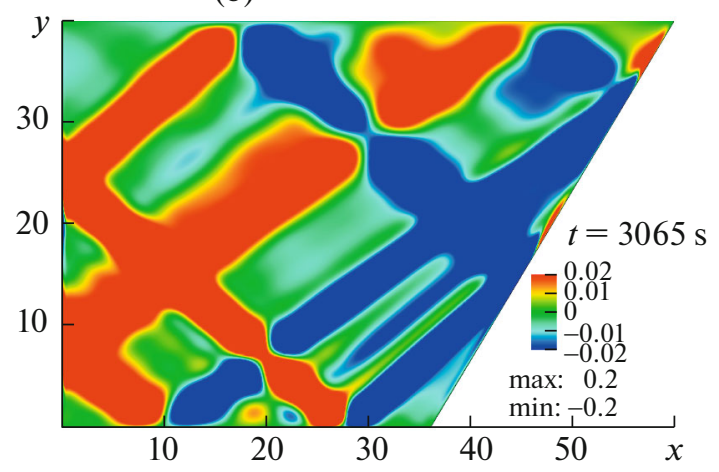

(d)

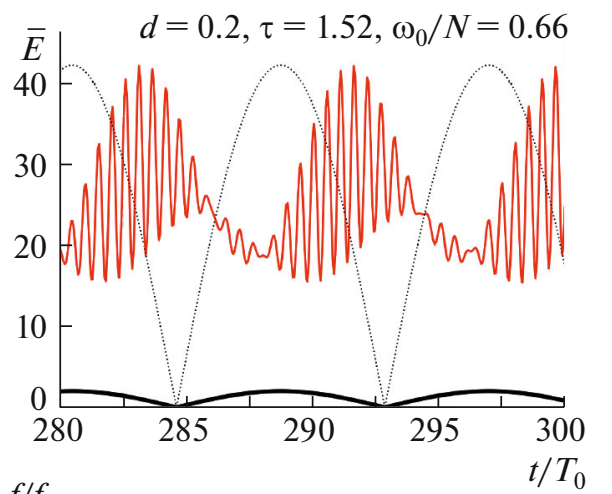

(f)

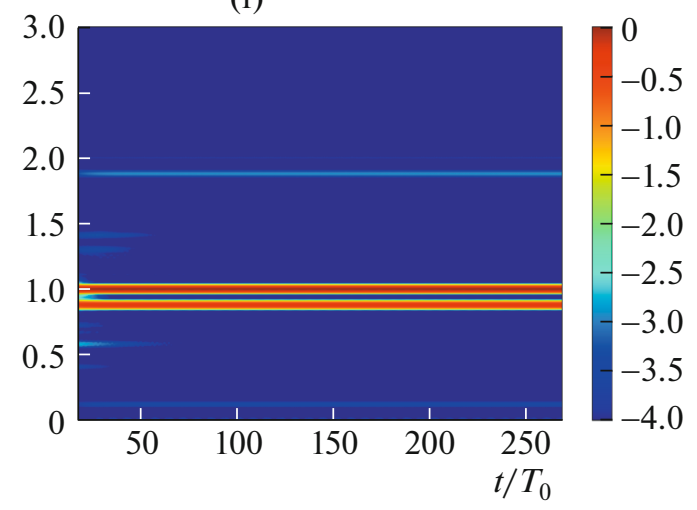

Fig. 4. Vertical velocity component $v_{y}$ (a) and pressure (b) in the biharmonic regime at the amplitudes $a=0.02 \mathrm{~cm} / \mathrm{s}$ and the corresponding vertical velocity component in the middle of the first attractor ray (c), kinetic energy (d), spectrum (e), and time-and-frequency diagram (f). Black curves in (c, d) show the envelopes of the wave-maker oscillation amplitude.

$\left(\omega_{2}-\omega_{1}\right) / N$; however, their value is less than the main peak by more than two orders of magnitude. The time instants corresponding to the maximum kinetic energy lag significantly behind the time instants corresponding to the maximum wave-maker oscillation amplitudes. Of importance also to note that when the system reaches the steady-state beating regime the average kinetic energy of the system excited by a biharmonic perturbation is equal to the sum of the energies of attractors exited by the monochromatic perturbations in isolation from one another $\bar{E}_{k}=21.7 \times 10^{-4} \approx \bar{E}_{k 1}+\bar{E}_{k 2}=(8.45+13.2) \times 10^{-4}=21.65 \times 10^{-4} \mathrm{erg} / \mathrm{cm}^{2}$. Thus, in the linear regime the linear superposition principle holds with high accuracy. This is also fulfilled at small frequency difference $\left(\omega_{1}-\omega_{2}\right) / N$.

\subsection{Nonlinear Regime}

In Figs. 5 and 6 we have reproduced the examples of nonlinear dynamics of wave attractors generated by wave-maker biharmonic oscillations for $\omega_{1} / N=0.66, \omega_{2} / N=0.628$, and $\delta \omega / N=0.031$ (Fig. 5) and 
(a)

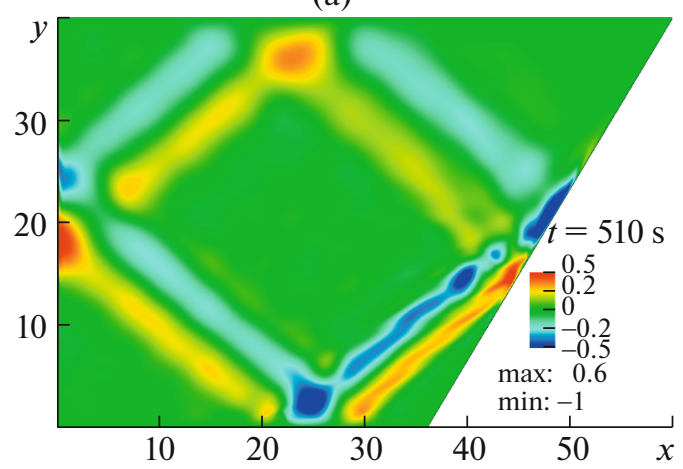

(c)

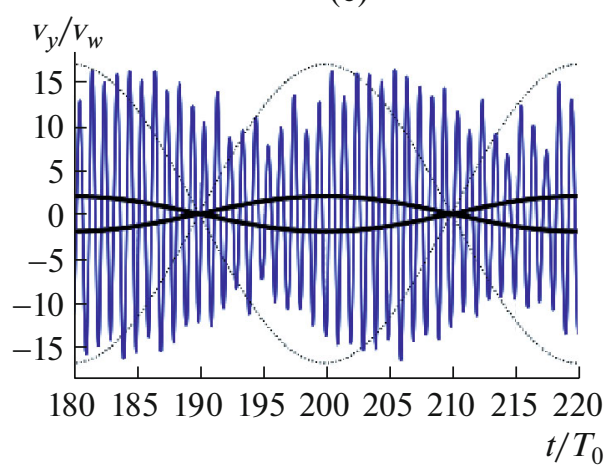

(e)

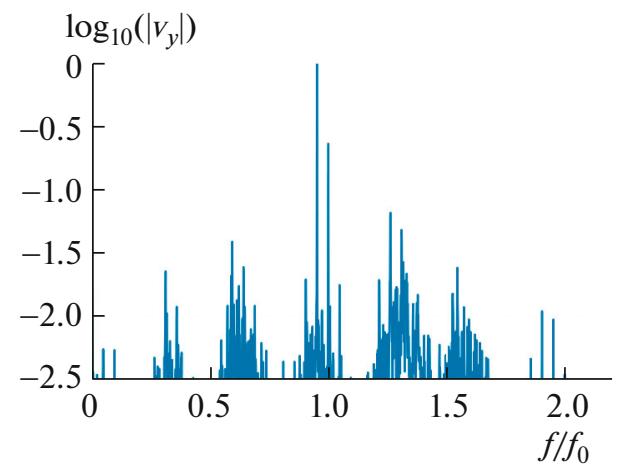

(b)

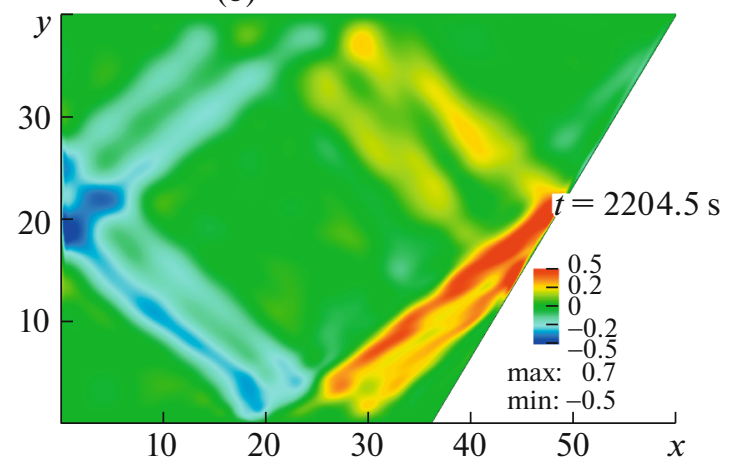

(d)
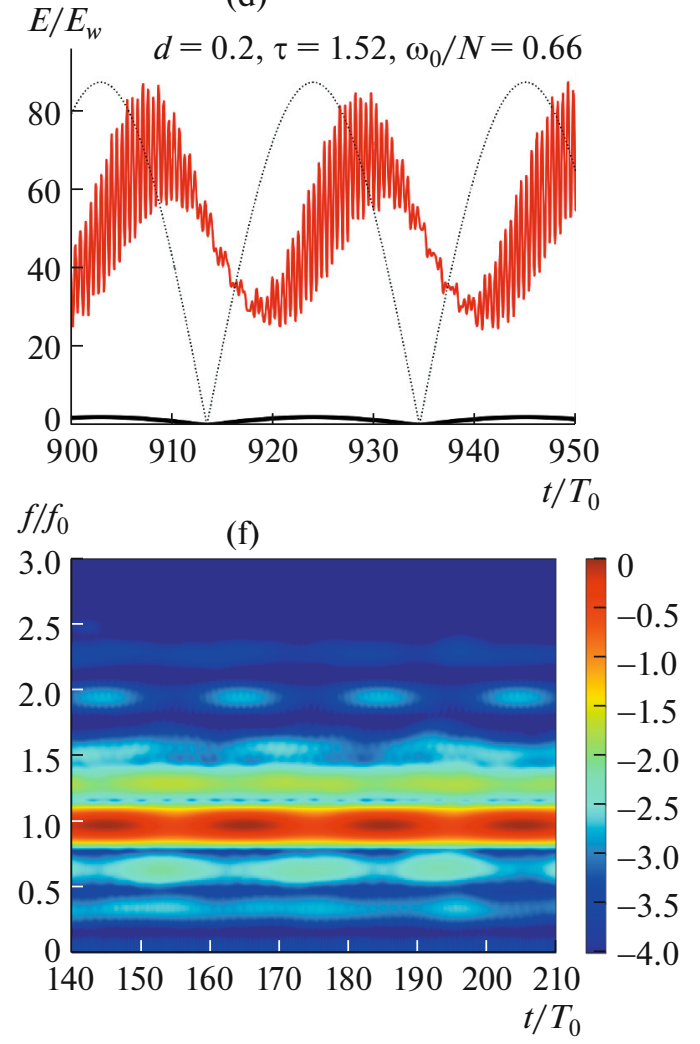

Fig. 5. Vertical velocity component in forming (a) and establishing (b) the biharmonic attractor with the amplitudes $a=$ $0.05 \mathrm{~cm} / \mathrm{s}$ and the relative frequency difference 0.05 and the corresponding vertical velocity (c), kinetic energy (d), spectrum (e), and time-and-frequency diagram (f). Black curves in (c, d) show the envelopes of the wave-maker oscillation amplitude.

$\omega_{1} / N=0.628, \omega_{2} / N=0.641$, and $\delta \omega / N=0.013$ (Fig. 6). In all the cases the wave-maker oscillation amplitudes were equal to $a_{1}=a_{2}=0.05 \mathrm{~cm}$. It can be seen that the wave motion with a complex frequency spectrum is formed in both cases, the trend to the more dense spectrum "population" being observed with decrease in the frequency mismatch $\delta \omega$. The distinctive beating process can be seen on the graphs of the vertical velocity as a function of time, but, in addition to mean energy oscillations, nontrivial dynamics of high-frequency energy fluctuations takes place, namely, the fluctuation amplitudes can differ by an order of magnitude in the phases of growth and decrease of the envelope of the wave-maker oscillation amplitude. Thus, the periodic "bursts" of wave turbulence are distinctive in the nonlinear biharmonic regime. Such "bursts" can clearly be seen on the time-and-frequency diagrams shown in Figs. 5 and 6. In particular, on the time-and-frequency diagram given in Fig. 5 we can see that at the frequency close to the frequency of the external forcing the signal amplitude "beatings" are shifted in time with respect to the "beat- 
(a)

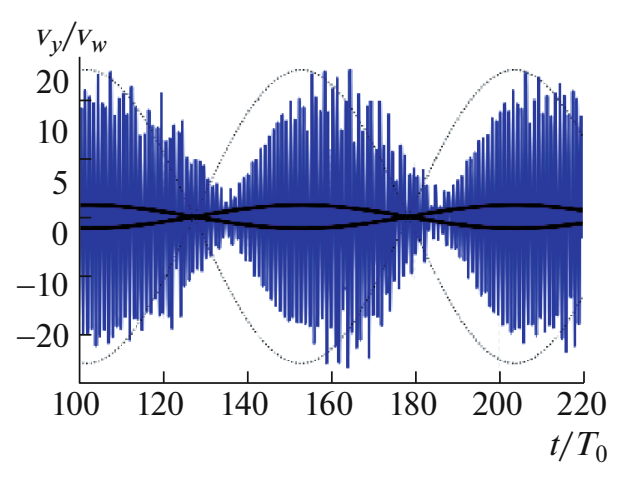

(c)

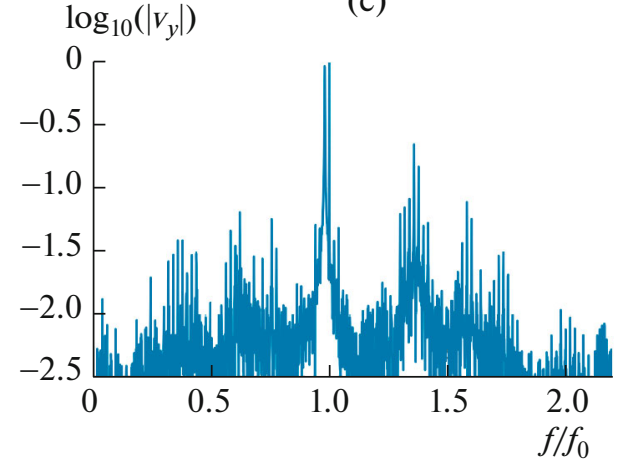

(b)
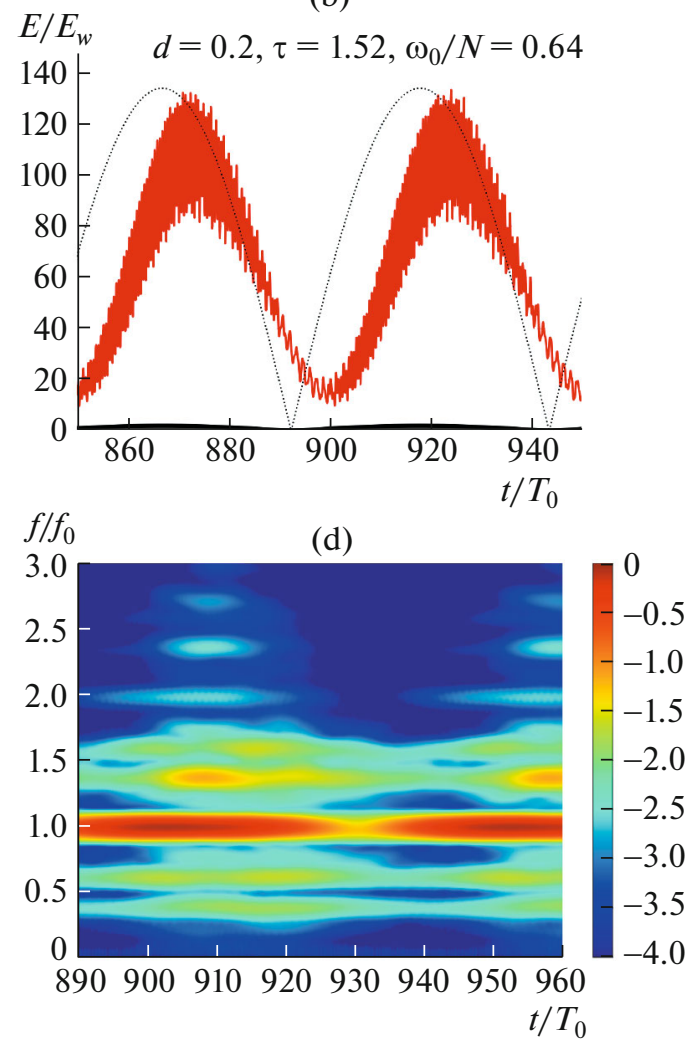

Fig. 6. Vertical velocity component (a), kinetic energy (b), spectrum (c), and time-and-frequency diagram (d) in the biharmonic regime with the amplitudes $a=0.05 \mathrm{~cm} / \mathrm{s}$ and the relative frequency difference 0.02 . Black curves in (a) and (b) show the envelopes of the wave-maker oscillation amplitude.

ings" of daughter waves. Thus, the "beatings" of the wave-maker oscillation envelope, the "beatings" of the mean kinetic energy, and the "bursts" of wave turbulence are mismatched in time. We can assume that in the natural systems there is a time mismatch between the envelope of the internal tide amplitude and the enhancement of internal wave turbulence and mixing. A preliminary investigation of the energy of attractors generated by the biharmonic perturbation shows that in the nonlinear case the mean energy of the system differs significantly from the sum of energies of the components.

\section{SUMMARY}

Dynamics of internal wave attractors under the external biharmonic forcing are first investigated. To get the intervals of parameters of greatest interest, the regimes under monochromatic forcing were first studied, and the frequency range was determined, where the attractor generation is the most effective. The investigation of the behavior of attractors under external biharmonic forcing showed that in the linear case the superposition principle is valid, namely, the attractors generated by each of the components of the biharmonic perturbation almost do not interact between themselves. In the nonlinear case, the beating regime accompanied by the bursts of wave turbulence developed as a result of a cascade of triad interactions can be observed under the external biharmonic forcing. In this case the level of kinetic energy fluctuations in the phase of growth of the envelope of the wave-maker oscillation amplitude can be by an order of magnitude higher than the level corresponding to decrease in the wave-maker oscillation amplitude.

\section{FUNDING}

The work was carried out under financial support from the Russian Science Foundation (RSF) under the grant no. 19-11-00169 using the equipment of the shared research facilities of HPC computing resources at Moscow State University and the computer complex of the shared research facilities of NRC Kurchatov Institute. 


\section{OPEN ACCESS}

This article is licensed under a Creative Commons Attribution 4.0 International License, which permits use, sharing, adaptation, distribution and reproduction in any medium or format, as long as you give appropriate credit to the original author(s) and the source, provide a link to the Creative Commons licence, and indicate if changes were made. The images or other third party material in this article are included in the article's Creative Commons licence, unless indicated otherwise in a credit line to the material. If material is not included in the article's Creative Commons licence and your intended use is not permitted by statutory regulation or exceeds the permitted use, you will need to obtain permission directly from the copyright holder. To view a copy of this licence, visit http://creativecommons.org/licenses/by/4.0/.

\section{REFERENCES}

1. Mowbray, D.E. and Rarity, B.S.H., A theoretical and experimental investigation of the phase configuration of internal waves of small amplitude in a density stratified liquid, J. Fluid Mech., 1967, pp. 1-16.

2. Dauxois, T. and Young, W., Near-critical refection of internal waves, J. Fluid Mech., 1999, pp. 271-295.

3. Maas, L., Wave attractors: linear yet non linear, Int. J. Bifurcation Chaos, 2005, pp. 2757-2782.

4. Lam, F. and Maas, L., Geometric focusing of internal waves, J. Fluid Mech., 1995, pp. 1-41.

5. Lam, F.P.A., Benielli, D., Sommeria, J., and Maas, L.R.M. Observations of an internal wave attractor in a confined stably stratified fluid, Nature, 1997, pp. 557-561.

6. Sibgatullin, I. and Ermanyuk, E., Internal and inertial wave attractors: a review, Journal of Applied Mechanics Technical Physics, 2019, pp. 284-302.

7. Morozov, E., Semidiurnial internal wave global field, Deep-Sea Res., 1995, pp. 135-148.

8. Garrett, K. and Kunze, E., Internal tide generation in deep ocean, Annu. Rev. Fluid Mech., 2007, pp. 57-87.

9. Tang, W. and Peacock, T. Lagrangian coherent structures and internal wave attractors, Chaos, 2010.

10. Echeverri, P., Yokossi, T., Balmforth, N.J., and Peacock, T., Tidally generated internal-wave attractors between double ridges, J. Fluid Mech., 2011, pp. 354-374.

11. Wang, G., Zheng, Q., Lin, M., and Qiao, F., Three dimensional simulation of internal wave attractors in the luzon strait, Acta. Oceanol. Sin., 2015, pp. 14-21.

12. Guo Y. and Holmes-Cerfon, M., Internal wave attractors over random, small-amplitude topography, J. Fluid Mech., 2016, pp. 148-174.

13. Haren, van H., Maas, L., and Gerkema, T., Patchiness in internal tidal beams, Journal of Marine Research, 2010, pp. 237-257.

14. Sutherland, B.R., Internal wave instability: Wave-wave vs wave-induced mean flow interactions, Phys. Fluids, 2006.

15. Dauxois, T., Joubaud, S., Odier, P., and Venaille, A., Instabilities of internal gravity wave beams, Annu. Rev. Fluid Mech., 2018, pp. 131-156.

16. Mercier, J.M., Garnier, N.B., and Dauxois, T., Reflection and diffraction of internal waves analyzed with the Hilbert transform, Physics of Fluids, 2008.

17. Providukhina, M. and Sibgatullin, I., Application of spectral data processing methods to the results of the numerical simulation of internal wave attractors, Proceedings of the Institute for System Programming of the RAS, 2016, pp. 275-282.

18. Grisouard, N., Staquet, C., and Pairaud, I., Numerical simulation of a two-dimensional internal wave attractor, J. Fluid Mech., 2008, pp. 1-14.

19. Brouzet, C., Dauxois, T., Ermanyuk, E., Joubaud, S., Kraposhin, M., and Sibgatullin, I., Direct numerical simulation of stratified-fluid internal wave attractors in a trapezoidal domain with oscillating vertical wall, Proceedings of the Institute for System Programming of the RAS, 2014, pp. 117-142.

20. Brouzet, C., Sibgatullin, I.N., Scolan, H., et al., Internal wave attractors examined using laboratory experiments and 3d numerical simulations, Journal of Fluid Mechanics, 2016, pp. 429-432.

21. Sibgatullin, I.N., Ermanyuk, E.V., Maas, L., et al., Direct numerical simulation of three-dimensional inertial wave attractors, in: 2017 Ivannikov ISPRAS Open Conference (ISPRAS), 2017, pp. 137-143.

22. Lam, F. and Maas, L., Internal wave focusing revisited; a reanalysis and new theoretical links, Fluid Dyn. Res., 2008, pp. 95-122.

23. Brouzet, C., Sibgatullin, I.N., Ermanyuk, E.V., et al., Scale effects in internal wave attractors, Physical Review Letters, 2017.

24. Brouzet, C., Ermanyuk, E.V., Joubaud, S., et al., Energy cascade in internal wave attractors, Europhysics Letters, 2016.

25. Brouzet, C., Ermanyuk, E.V., Joubaud, S., et al., Internal wave attractors: different scenarios of instability, Journal of Fluid Mechanics, 2017, pp. 544-568.

26. Hazewinkel, J., Grisouard, N., and Dalziel, S., Comparison of laboratory and numerically observed scalar fields of an internal wave attractor, Eur. J. Mech. B Fluids, 2011, pp. 51-56.

27. Fischerand, P. and Ronquist, E., Spectral element methods for large scale parallel Navier-Stokes calculations, Comput. Math. Appl. Mech. Engng., 1994, pp. 69-76.

Translated by E.A. Pushkar 